\title{
Transcranial magnetic stimulation devices for biphasic and polyphasic ultra-high frequency protocols
}

\author{
Gattinger $\mathrm{N}^{1}$, Jung $\mathrm{NH}^{2}$, Mall $\mathrm{V}^{3}$ and Gleich $\mathrm{B}^{4 *}$ \\ ${ }^{1}$ Munich School of Bioengineering (MSB), Technical University of Munich, Munich, Germany \\ ${ }^{2}$ School of Medicine, Social Pediatrics, Technical University of Munich, Munich, Germany \\ ${ }^{3}$ School of Medicine, Social Pediatrics, Technical University of Munich and with kbo Kinderzentrum München, Munich, Germany \\ ${ }^{4}$ Munich School of Bioengineering (MSB), Technical University of Munich, Munich, Germany
}

\begin{abstract}
Objective: This paper describes the development of ultra-high frequency transcranial magnetic stimulators for biphasic and polyphasic quadripulse stimulation (QPS) of the human cortex in order to investigate neural plasticity. QPS originally describes a set of four monophasic stimuli with an inter-stimulus-interval (ISI) in the range of 1.5 to $100 \mathrm{~ms}$, typically repeated every five seconds. Besides QPS, the described devices are able to set a quadripulse at much higher repetition rates (IBIs down to $200 \mathrm{~ms}$ ) enabling quadri-Theta-Burst-Stimulation (qTBS).

Methods: Insulated gate bipolar transistors (IGBT) were used as high voltage/high speed switch to control the current flowing through the stimulation coil. Biphasic and the polyphasic stimuli were applied over the human primary motor cortex (M1-HAND) to elicit motor evoked potentials (MEP). Technically parameters of the devices were determined and applicability for qTBS proto-cols is derived.
\end{abstract}

Results: We built two devices, which allow the application of quadripulses with biphasic and polyphasic pulse currents, respectively. ISI as short as 1.0 ms with a IBI down to $200 \mathrm{~ms}$ is possible. In addition the devices allow to apply user defined protocols enabling individualized treatment.

Conclusion: The biphasic as well as the polyphasic qTBS device is able to clearly elicit MEPs and is strong enough to apply qTBS protocols to subjects.

Significance: This paper describes a novel topology of a stimulation device, which enables QPS and qTBS with biphasic or polyphasic pulse shapes at ultra-high frequencies. This enables research on protocols that can modulate cortico-spinal excitability within short stimulation times.

\section{Introduction}

Repetitive transcranial magnetic stimulation (rTMS) - like thetaburst stimulation (TBS) [1,2] or QPS [3] - is one of the most popular methods for non-invasive brain stimulation in humans and becomes gradually more noticed in basic neuroscience as well as in the treatment of various neurological diseases such as drug resistant depression and re-habilitation after stroke [2]. The first TMS device, introduced by Barker et al. in 1985 [4], has a monophasic pulse shape. Briefly, in TMS a current pulse, flowing through a stimulation coil generates a pulsed magnetic field, which in turn induces a pulsed electric field inside the brain. This induced electric field depolarizes the neuron and thus evokes an action potential. To generate a pulse current the stimulation coil and a pulse capacitor build up a resonant circuit. Before a stimulus can be applied, the pulse capacitor, which acts as energy storage, has to be charged to a certain voltage level which corresponds to the current and thus to the pulse energy. Once the capacitor is charged, the system is ready for releasing a stimulus. Therefore, the application coil is connected directly via a high voltage switch to the capacitor and as soon as this switch is closed a sinusoidal current flow-through the coil starts immediately. In monophasic devices the system is damped with a power resistor and a shunting diode after a quarter of the oscillation period. Because of this the current flow through the application coil, falls smoothly back to zero. After each stimulus the pulse capacitor has to be completely recharged. This is the reason why commercial monophasic magnetic stimulators are limited to less than five pulses per second.
Cadwell et al. first introduced polyphasic stimulators in 1990 [5]. These devices do not damp the coil current after the first quarter of the oscillation period and therefore a sinusoidal oscillation of the current occurs. As the coil current repeatedly changes its direction and thus the induced electric field also has repeatedly different direction, the pulse shape is called polyphasic. The polyphasic device, introduced by Cadwell et al. has a heavy damping due to parasitic ohmic losses mainly of the application coil, the wiring, the high voltage switch and also the capacitor. Biphasic stimulators interrupt the current oscillation after the first period by opening the switch. The interruption of the oscillation after one period can be achieved easily by using thyristors as high voltage switches. These components block automatically, when the current through them is zero. The great benefit of a biphasic stimulator is that most of the deployed energy is fed back into the pulse capacitor and thus can be used again for the next stimulus. Nowadays biphasic stimulators have smaller damping characteristic than the Cadwell device, as electronic components get better, but also underlie damping

Correspondence to: Bernhard Gleich, Munich School of Bioengineering (MSB), Technical University of Munich, Munich, Germany. E-mail: gleich@tum.de

Key words: biphasic, monophasic, polyphasic, transcranial magnetic stimulation, TMS, repetitive TMS, non-invasive, quadripulse stimulation, QPS, qTBS

Received: February 03, 2018; Accepted: February 25, 2018; Published: February 28,2018 
effects due to parasitic ohmic losses on all power components involved. This loss of energy has to be recharged before the next stimuli can be set. But as most of the pulse energy can be used for the next stimulus repetition rates of biphasic magnetic stimulators nowadays reach up to 100 pulses per second.

Today, many different TMS protocols are known. They can be divided into continuous or intermittend (patterned) protocols. Examples of these protocols are high or low frequency rTMS and continuous or intermittend theta-burst stimulation (iTBS, cTBS, respectively) [1]. All of these protocols are based on biphasic stimulators. Since monophasic stimulators allow the connection of several devices with one stimulation coil, new stimulation protocols where developed for the monophasic pulse shape as well. By connecting two or more monophasic stimulators to one coil, two or more stimuli can be released shortly after each other (down to $1.0 \mathrm{~ms}$ delay between two pulses) and thus enable neurophysiologically driven paired pulse TMS protocols, for instance.

Inspired by tetanic stimulation protocols, which are often used in animal experiments and induce robust long-term potentiation (LTP), which is considered to be the neuro-physiological correlate for learning and memory [6], Hamada et al. built a set-up consisting of four monophasic devices, which were connected to one single application coil [3]. One train consisted of four stimuli with the same intensity and an ISI of $1.5 \mathrm{~ms}$ and was repeatedly given up to $0.2 \mathrm{~Hz}$ (one quadripulse every five seconds). They found that this QPS induced long-lasting locally restricted facilitation or inhibition of motor cortical excitability, depending on the repetition rate of the quadripulses $[3,6]$.

In contrast to TBS where commonly biphasic stimuli are used, QPS is in an early stage of development and most QPS research is done with monophasic pulses as introduced by Hamada et al. [3]. There are some evidences that monophasic and biphasic pulses stimulate interneurons differently or that they even activate a different subset of interneurons all together [8]. Pell et al. indicated that biphasic and monophasic pulse shapes could activate neural circuits to a different degree $[9,10]$. Thus, identical protocols given with monophasic or biphasic pulses may lead to different results. In 2016 Nakarumara et al. compared monophasic and biphasic QPS by connecting four biphasic or monophasic stimulation devices to one stimulation coil, respectively [7]. Highest repetition rate used was one quadripulse every 2.5 seconds. When one quadripulse was given every 5 seconds, this protocol showed comparable results, but biphasic QPS had shorter lasting after-effects compared with monophasic QPS [7].

To enable further research on biphasic QPS we present a TMS device that is able to release QPS protocols with biphasic pulse shape and repetition rates up to $1000 \mathrm{~Hz}$ (ISI down to $1.0 \mathrm{~ms}$ ). In contrast to the described set-up in [7], we use a single device that is able to release a quadripulse every $200 \mathrm{~ms}$. Because of the short IBI time of $200 \mathrm{~ms}$, we call this kind of protocol quadri- TBS (qTBS). It was also shown that a polyphasic pulse consisting of two full-sine cycles reduces the energy needed for a certain MEP response compared to a biphasic pulse with the same oscillation frequency [11]. Referring to this finding, we also describe a second TMS device that is able to apply the above mentioned qTBS protocols with polyphasic (double sine-wave) pulses.

\section{System description}

Compared to conventional TMS devices some opti-mizations of the power circuitry have to be done to be able to apply biphasic and polyphasic qTBS protocols. This includes the high voltage power supply, the pulse switch as well as the control unit of the stimulator. Figure 1 shows a simplified schematic of the power circuitry, which is used for the biphasic and for the polyphasic device. Table 1 as well as Table 2 displays the main power train components of the biphasic device and the polyphasic device, respectively.

\section{Power supply and pulse capacitor}

The biphasic qTBS device has a high voltage power supply that charges the energy storage capacitor constantly to $2,250 \mathrm{~V}$. The pulse capacitor C3 is charged to the desired level via Q1 and R1 as well as via Q4 and R4. The pulse capacitor can be discharged by Q2, Q3, R2 and R3. Discharging takes less than half of the charging time since the energy storage capacitor is connected with reversed polarity to the pulse capacitor. In case of an emergency, e.g. disconnection of the coil during stimulation, all charging IGBTs are switched on. This ensures a fast reduction of the high voltage of both, the energy storage capacitor and the pulse capacitor. To reduce the reverse voltage, the charging IGBTs have to resist when a biphasic pulse is released both the energy storage capacitors $\mathrm{C} 1$ and $\mathrm{C} 2$, as well as the pulse capacitor $\mathrm{C} 3$ are balanced and work to the same virtual ground. The pulse capacitor of the biphasic qTBS device can be adjusted in steps of $11 \mathrm{~V}$ in the range of zero to $2,200 \mathrm{~V}$, which leads to a stimulation accuracy of $0.5 \%$ of maximum stimulator output (MSO).

The polyphasic qTBS device has a high voltage power supply that charges the energy storage capacitor constantly to $2,800 \mathrm{~V}$. The pulse capacitor is charged to a maximum of $2,700 \mathrm{~V}$. Charging and discharging works the same as for the previously described biphasic qTBS device. The stimulation accuracy for the polyphasic qTBS device is $0.5 \% \mathrm{MSO}$ as well.

\section{Pulse switch and IGBT-driver}

In contrast to conventional biphasic or monophasic TMS devices that mostly use thyristors as high power switches, we used a high voltage power IGBT. This allows the release of stimuli very close to each other and with short oscillation periods. For the use in a biphasic or polyphasic TMS device, the crucial parameter of a thyristor is the so called turn-off time tq, which is defined as the time between the instant anode current becomes zero and the instant SCR regains forward blocking capability. Being able to block the capacitor voltage at the end of the pulse, tq has to be shorter than a half of the oscillation period. Second crucial parameter is the maximum rating of the current rise (max. dI/dt). As no power thyristor with suitable tq and sufficient dI/ $\mathrm{dt}$ rating was available for the polyphasic qTBS device, we decided to use a power IGBT. In need to develop a driver circuitry for the IGBT anyway, we decided to use the same components in the biphasic qTBS device, too.

During the first half-sine wave (positive current flow through the coil L1) the IGBT Q5 is conducting, whereas during the second half-wave (negative current flow through the coil) the included freewheeling diode is conducting. In this circuit topology, the IGBT may never be shut off when it is under load, as the coil-induced voltage would inevitably damage the IGBT. In normal use the IGBT is only shut off when the free-wheeling diode is under load.

The implemented IGBTs have a repetitive peak collector load of $2,000 \mathrm{~A}$. Since the maximum peak current flow of the biphasic device is up to 5,200 A the specified current ratings are exceeded by a factor of approximately 2.5 . This overload is tolerable due to the expedient duty cycle [12-14]. For a worst-case consideration we can assume a qTBS protocol with 100 quadripulses per second, which leads to 400 pulses 


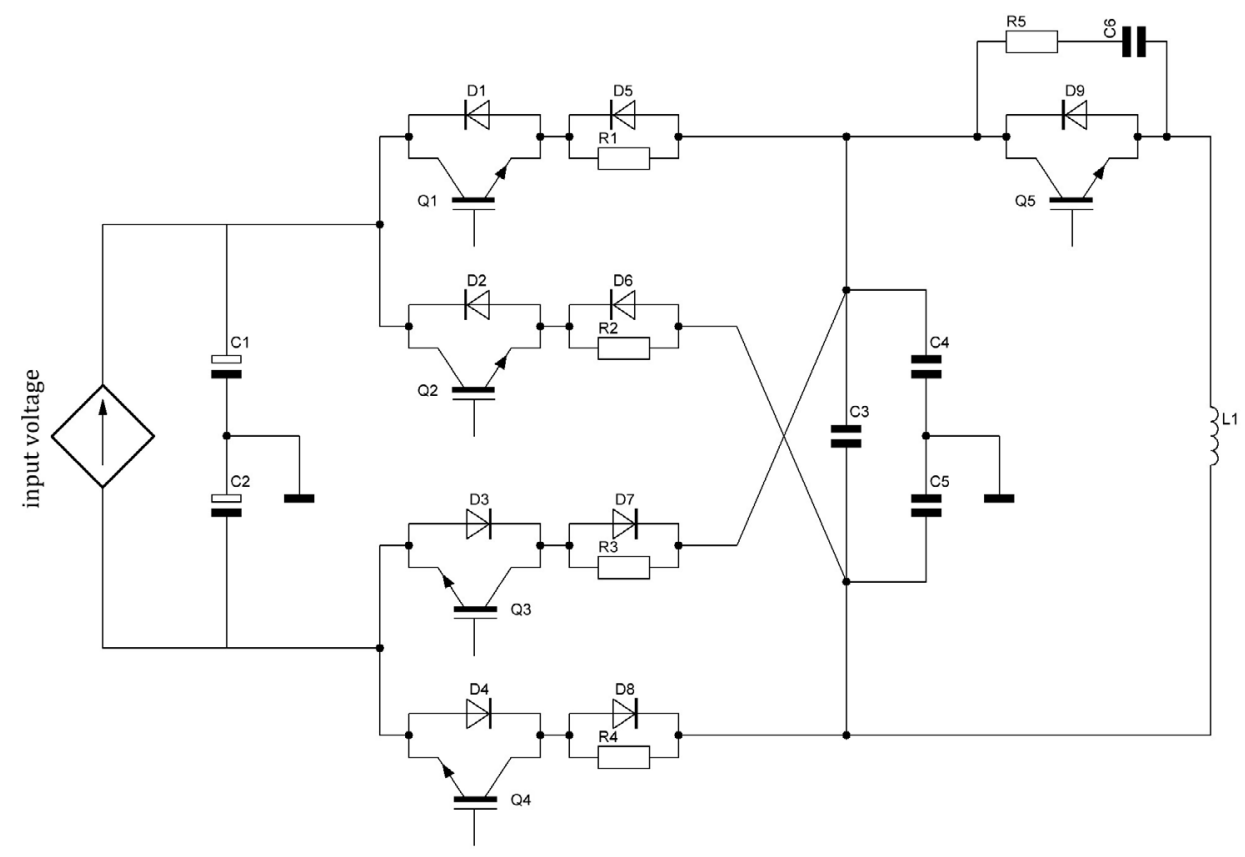

Figure 1. Simplified schematic of the biphasic and polyphasic qTBS device, respectively.

Table 1. Main Power Train Components of The Biphasic qTBS Device

\begin{tabular}{|c|c|c|c|c|}
\hline Component & Assignment & Nominal Rating & Part \# & Manufacturer \\
\hline \multirow[t]{2}{*}{$\mathrm{C} 1, \mathrm{C} 2$} & \multirow[t]{2}{*}{ Energy Storage } & $\begin{array}{l}6 \mathrm{x} 680 \mu \mathrm{F} \pm 10 \% ; 450 \mathrm{VDC} \text { (series } \\
\text { and parallel); }\end{array}$ & \multirow{2}{*}{ MAL215757681E3 } & \multirow[t]{2}{*}{ Vishay } \\
\hline & & total capacitance: $\sim 454 \mu \mathrm{F}$ & & \\
\hline $\mathrm{C} 3$ & Pulse Capacitor & $\begin{array}{c}3 \times 22 \mu \mathrm{F} \pm 10 \% ; 3,000 \mathrm{VDC} \text { (in } \\
\text { parallel) }\end{array}$ & KMKP 1400-22IB & Vishay \\
\hline $\mathrm{C} 4, \mathrm{C} 5$ & Symmetry Capacitors & $470 \mathrm{nF} \pm 10 \% ; 1,600 \mathrm{VDC}$ & RBPS & Acrovox \\
\hline C6 & Snubber Capacitor & $270 \mathrm{nF} \pm 10 \% ; 2,500 \mathrm{VDC}$ & MMKP 386 & Vishay \\
\hline $\mathrm{Q} 1-\mathrm{Q} 4$ & Charging IGBTs & 4,000 VDC; $40 \mathrm{~A}$ & IXEL40N400 & IXYS \\
\hline Q5 & Power IGBT & $3,300 \mathrm{VDC} ; 1,000 \mathrm{~A}$ & FZ1000R33HE3 & Infineon \\
\hline D1 - D4 & Free-Wheeling Diode & $4,000 \mathrm{VDC} ; 0.25 \mathrm{~A}$ & GP02-40 & Vishay \\
\hline \multirow{2}{*}{ D5 } & \multirow{2}{*}{ Free-Wheeling Diode } & \multirow{2}{*}{$3,300 \mathrm{VDC} ; 1,000 \mathrm{~A}$} & FZ1000R33HE3 (included & \multirow{2}{*}{ Infineon } \\
\hline & & & in the IGBT module) & \\
\hline $\mathrm{D} 6-\mathrm{D} 9$ & Free-Wheeling Diode & 2x 1,000 VDC; 3 A (in series) & $1 \mathrm{~N} 5408$ & Diodes Inc. \\
\hline $\mathrm{R} 1, \mathrm{R} 4$ & Charging Resistors & $2 \times 12 \mathrm{Ohm} \pm 1 \%$ (in series) & HS100 & Arcol \\
\hline R2, R3 & Discharging Resistors & $2 \times 22 \mathrm{Ohm} \pm 1 \%$ (in series) & HS100 & Arcol \\
\hline R5 & Snubber Resistor & $6.8 \mathrm{Ohm} \pm 1 \%$ & HS100 & Arcol \\
\hline
\end{tabular}

Table 2. Main Power Train Components of The Polyphasic qTBS Device

\begin{tabular}{|c|c|c|c|c|}
\hline Component & Assignment & Nominal Rating & Part \# & Manufacturer \\
\hline \multirow[t]{2}{*}{$\mathrm{C} 1, \mathrm{C} 2$} & \multirow[t]{2}{*}{ Energy Storage } & $\begin{array}{l}8 \times 680 \mu \mathrm{F} \pm 10 \% ; 450 \mathrm{VDC} \text { (series } \\
\text { and parallel); }\end{array}$ & \multirow[t]{2}{*}{ MAL215757681E3 } & \multirow[t]{2}{*}{ Vishay } \\
\hline & & total capacitance: $\sim 340 \mu \mathrm{F}$ & & \\
\hline $\mathrm{C} 3$ & Pulse Capacitor & $\begin{array}{c}2 \times 6.8 \mu \mathrm{F} \pm 10 \% ; 3,000 \mathrm{VDC} \text { (in } \\
\text { parallel) }\end{array}$ & KMKP 1400-6.8IB & Vishay \\
\hline $\mathrm{C} 4, \mathrm{C} 5$ & Symmetry Capacitors & $470 \mathrm{nF} \pm 10 \% ; 2,000 \mathrm{VDC}$ & KMKP 386 & Vishay \\
\hline C6 & Snubber Capacitor & 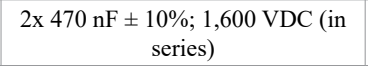 & RBPS & Acrovox \\
\hline $\mathrm{Q} 1-\mathrm{Q} 4$ & Charging IGBTs & $4,000 \mathrm{VDC} ; 40 \mathrm{~A}$ & IXEL40N400 & IXYS \\
\hline Q5 & Power IGBT & $3,300 \mathrm{VDC} ; 1,000 \mathrm{~A}$ & FZ1000R33HE3 & Infineon \\
\hline $\mathrm{D} 1-\mathrm{D} 4$ & Free-Wheeling Diode & $4,000 \mathrm{VDC} ; 0.25 \mathrm{~A}$ & GP02-40 & Vishay \\
\hline \multirow{2}{*}{ D5 } & \multirow{2}{*}{ Free-Wheeling Diode } & \multirow{2}{*}{$3,300 \mathrm{VDC} ; 1,000 \mathrm{~A}$} & FZ1000R33HE3 (included & \multirow{2}{*}{ Infineon } \\
\hline & & & in the IGBT module) & \\
\hline D6 - D9 & Free-Wheeling Diode & $2 \times 1,000$ VDC; 3 A (in series) & 1N5408 & Diodes Inc. \\
\hline $\mathrm{R} 1-\mathrm{R} 4$ & Charging Resistors & $2 \times 33 \mathrm{Ohm} \pm 1 \%$ (in series) & HS100 & Arcol \\
\hline R5 & Snubber Resistor & $6.8 \mathrm{Ohm} \pm 1 \%$ & HS100 & Arcol \\
\hline
\end{tabular}


per second. The biphasic qTBS device has a pulse duration of about 160 $\mu$ s. Thus, in this case we have an averaged pulse pause of $2.34 \mathrm{~ms}$ and a duty cycle of 1:14.6 for $100 \%$ MSO. But we assume qTBS protocols to be applied at a sub-threshold level and will not be applied with $100 \%$ MSO. The polyphasic qTBS device works with lower pulse duration as well as lower peak currents and therefore the IGBT current stress is even lower than in the biphasic stimulator.

Compared to the current ratings, the specified peak voltage of the IGBT may never exceed the limit to avoid damage of the component. Particularly the polyphasic device works with a pulse capacitor voltage of up to $2,700 \mathrm{~V}$. That is very close to the maximum collector-emitter voltage of $3,300 \mathrm{~V}$ the IGBT can sustain. Therefore, the snubber circuitry consisting of R5 and C6, has to be designed very carefully.

The application of commercial IGBT drivers is not recommendable, since on the one hand, the working voltage level is close to the specified maximum and the current flow exceeds the manufacturers rating. On the other hand, the duty cycle is very advantageous. To reduce IGBTpower losses, the used custom made IGBT driver can turn on the IGBT within $1.2 \mu$ s and ensures a low on-resistance due to a gate voltage of $+18 \mathrm{~V}$. As the IGBT in normal operational mode is only turned off when the free-wheeling diode is under load the IGBT driver turns off the IGBT within $10 \mu$ s with a gate voltage of $-12 \mathrm{~V}$. Furthermore, the drive rmeasures the gate voltage of the IGBT to verify that it is properly turned on and off. In case a failure is detected the implemented microcontroller decides whether one has to wait for the diode-load period or if the IGBT can be shut off immediately.

\section{Control unit}

In the current implementation each device has four main modes. One is the classical rTMS mode where single pulses as well as known rTMS protocols like TBS can be applied with repetition rates up to 100 $\mathrm{Hz}$ (at 70\% MSO). Trigger-In functionality allows setting own rTMS protocols.

The second mode is a paired pulse mode, that allows setting two stimuli with different intensity and an ISI down to $1.0 \mathrm{~ms}$. The achievable delta in intensity from stimulus one to stimulus two depends on the chosen ISI and is for instance $-28 \%$ MSO to $+12 \%$ MSO (stimulus one: $65 \% \mathrm{MSO}$ ) at an ISI of $3.0 \mathrm{~ms}$. Repetition rates up to 5 $\mathrm{Hz}$ are supported. All pulse parameters can be adjusted by just using the front panel of the device. Through a external trigger-In event a paired pulse is released.

Third, a mode can be chosen where predefined qTBS/QPS protocols can be selected. Table 3 shows the active set of 9 selectable protocols and its intensity limits. Each protocol is defined by the following parameters: the number of stimuli to be applied in one train, the ISI, the repetition rate or rather the inter-burst-interval (IBI) as well as the total amount of stimuli to be applied. All parameters mentioned above are fixed the only degree of freedom is the stimulus intensity. No trigger-In functionality is possible in this mode, but it can be seen as an easy to use mode to set 'standard' QPS or qTBS protocols.

We called the fourth mode, which indeed is the most powerful one, individualized qTBS mode (iqTBS). This mode essentially needs a computer connected via USB-port to the stimulation device. By our custom made software Artemis running on personal computers using Microsoft Windows ${ }^{\text {tw }}$ XP or higher as an operating system, all protocol parameters as well as trigger-In and trigger-Out functionality can be configured. All timing parameters like ISI, IBI and Inter-Train-Interval (ITI) thereby can be adjusted in a step width of $100 \mu$ s. All protocol
Table 3. Example Set of Applyable qTBS and QPS Protocols

\begin{tabular}{|c|c|c|c|c|c|}
\hline ISI in ms & IBI in ms & $\begin{array}{c}\text { Stimuli in } \\
\text { total }\end{array}$ & $\begin{array}{c}\text { Total } \\
\text { duration in s }\end{array}$ & $\begin{array}{c}\text { max. MSO } \\
\text { biphasic } \\
\text { device }\end{array}$ & $\begin{array}{c}\text { max. MSO } \\
\text { polyphasic } \\
\text { device }\end{array}$ \\
\hline 1.5 & 200 & 1,440 & 72 & $65 \%$ & $75 \%$ \\
\hline 1.5 & 1,000 & 1,440 & 360 & $75 \%$ & $80 \%$ \\
\hline 1.5 & 5,000 & 1,440 & 1,800 & $75 \%$ & $80 \%$ \\
\hline 5 & 200 & 1,440 & 72 & $75 \%$ & $80 \%$ \\
\hline 5 & 1,000 & 1,440 & 360 & $80 \%$ & $85 \%$ \\
\hline 5 & 5,000 & 1,440 & 1,800 & $90 \%$ & $85 \%$ \\
\hline 50 & 200 & 1,440 & 72 & $95 \%$ & $85 \%$ \\
\hline 50 & 1,000 & 1,440 & 360 & $95 \%$ & $90 \%$ \\
\hline 50 & 5,000 & 1,440 & 1,800 & $95 \%$ & $90 \%$ \\
\hline
\end{tabular}

Nomenclature referred to Hamada et al. [3].

defining parameters can be stored and readjusted later on. For instance starting from a 'standard' qTBS protocol listed in Table 3 the fixed ISI that is used from one stimulus to another within a qadripulse (burst), can be replaced by individual ones. That, inter alia, enables one to set stimuli in a before measured I-wave latency, which might boost the effect of influencing neural plasticity [15]. Figure 2 visualizes an example set of four biphasic stimuli that can be repeated down to an IBI of $200 \mathrm{~ms}$. Once defined, the protocol settings are transferred to the stimulation device and evaluated by it. If valid parameters are loaded, they stay in the device as long as it is powered up or the parameters are replaced by newer ones. While stimulating, the device only works with these afore internally stored parameters. For safety reasons, the protocol parameters cannot be altered when the protocol is applied at the same moment. Being able to set trigger-In functionality to only a distinct stimuli (e.g. stimuli one of each burst) or may be even set to all stimuli within the protocol, we offer a maximum amount of flexibility. This makes the device perfect for closed loop experiment designs, too $[16,17]$.

\section{Coil current and induced voltage}

For each device type we recorded the coil current as well as the electric field with a connected Figure 8 air coil (P/N 510519, MAG \& More GmbH, Munich, Germany) with an inductance of $10 \mu \mathrm{H}$. The electric field was measured with a custom-made search (pick-up) coil consisting of a 5-turn circular winding with an inner and outer diameter of 0.5 and $2 \mathrm{~cm}$, respectively. The coil current was recorded with a Rogowski current probe (CWT60B, Power Electronics Measurements Ltd. Nottingham,UK). Both devices were set to $100 \% \mathrm{MSO}$ and a single pulse was released (Figure 3).

\section{Experimental results}

As proof of concept experiments a biphasic and a polyphasic qTBS device was used to determine resting motor threshold (RMT) as well as active motor threshold (AMT) by releasing stimuli over the M1-HAND. These determined thresholds should give us a forecast, whether the device output power is sufficient to apply qTBS protocols to subjects. Furthermore the effectiveness of the technical system was investigated.

\section{Subjects}

Four subjects participated in the preliminary experiment (mean age: $25.0 \pm 7.4$ years, two males, two females, all right handed). The study was carried out according to the Decleration of Helsinki and approved by the local Ethics Committee (vote 5423/12). All subjects were familiar with TMS, had no history of brain disease and fulfilled no exclusion criteria for TMS [18]. 

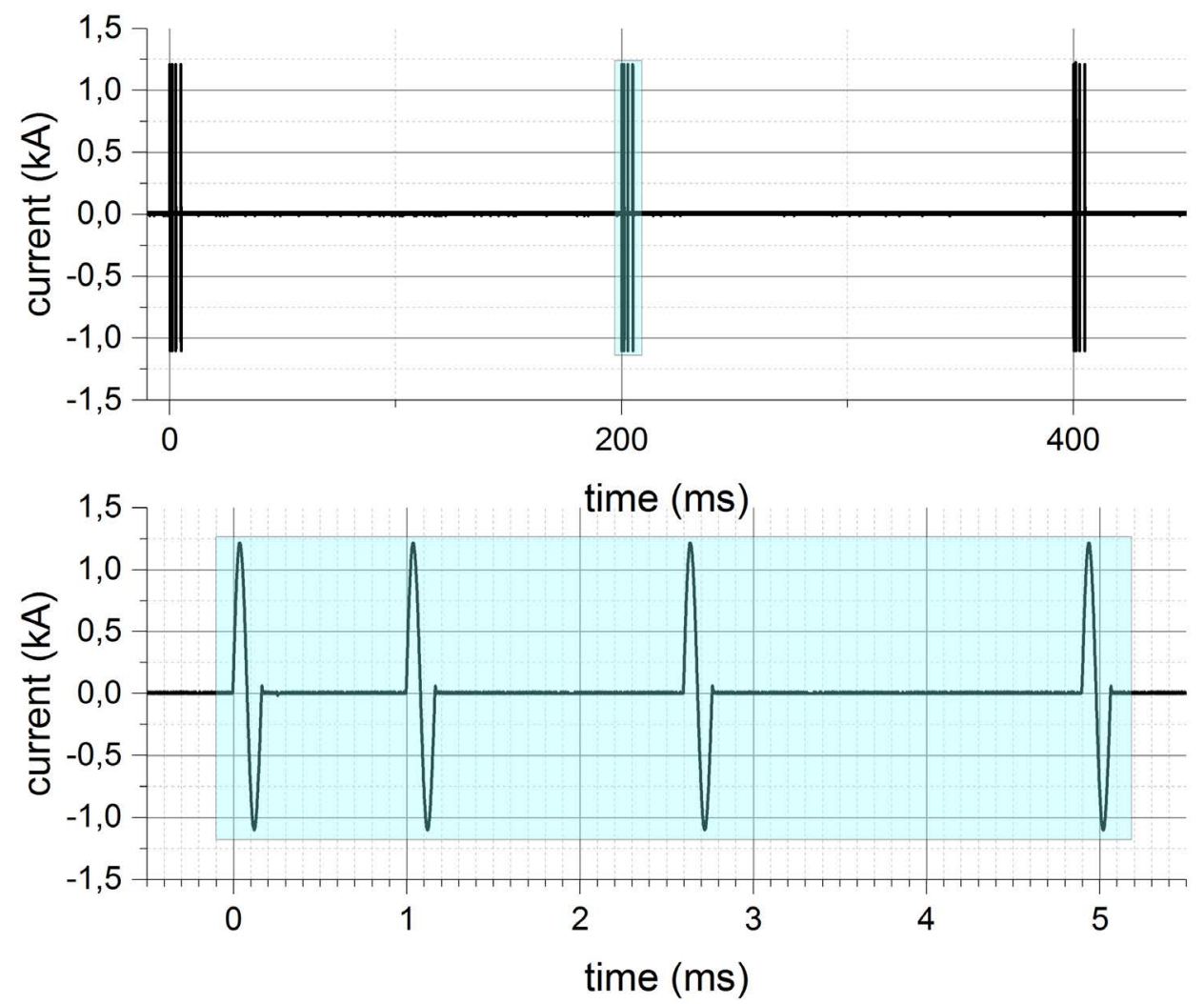

Figure 2. Example of an individualized qTBS (iqTBS) protocol setting with ISIs from $1.0 \mathrm{~ms}$ to $2.0 \mathrm{~ms}$ within a quadripulse (burst), IBI was chosen to be $200 \mathrm{~ms}$. Stimulator output power was set to $20 \% \mathrm{MSO}$.

a)

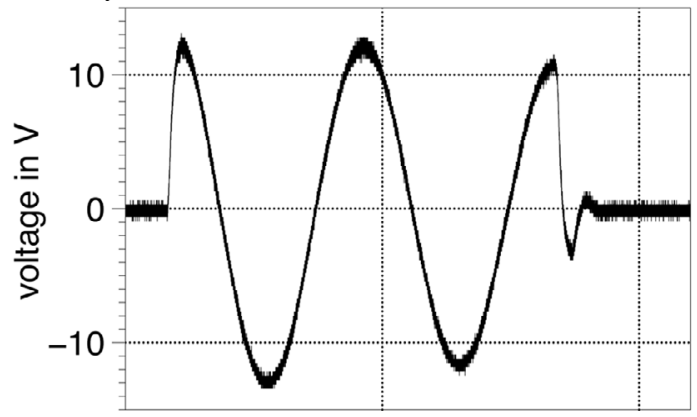

b)

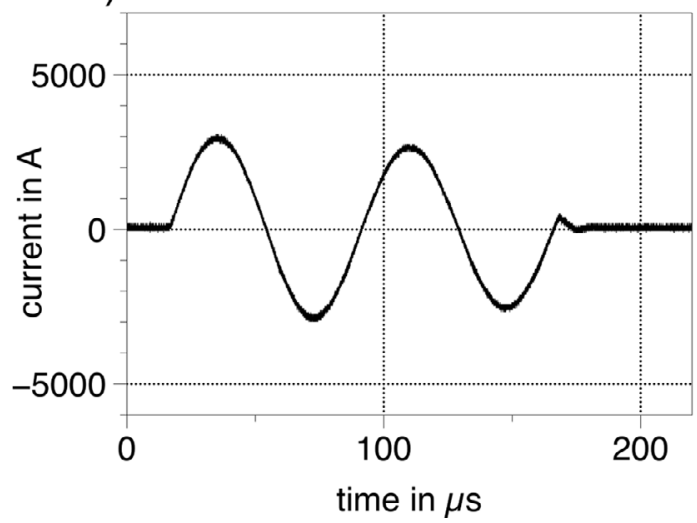

c)

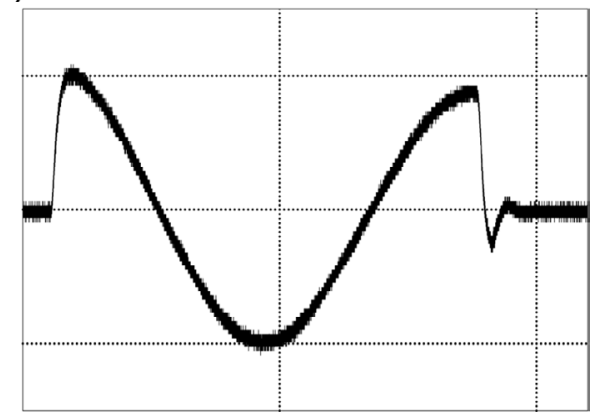

d)

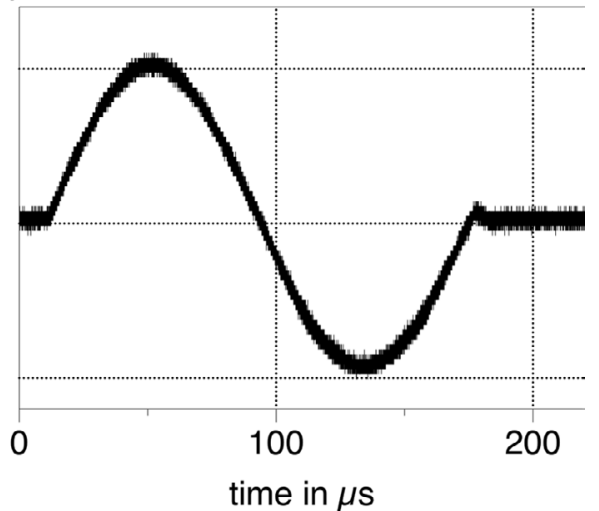

Figure 3. Induced electric field as well as coil current of the polyphasic qTBS device (inlay a and b) and the biphasic qTBS device (inlay c and d). Both pulses were applied at $100 \%$ MSO. 


\section{Experimental design}

To avoid carry-over effects, the order in which the different pulse shapes (biphasic and polyphasic) were presented was randomized. After RMT and AMT were determined with one device, a minimum intersession period of one week was hold till the threshold hunting session was done with device two.

A commercial Figure 8 shaped air coil (P/N 510519, MAG \& More $\mathrm{GmbH}$, Munich, Germany) was connected to the device. For both devices the initial current flow within the coil induced a current in the brain flowing from postero-lateral to antero-medial across the motor strip. MEPs were recorded by surface electromyography (EMG) from the non-dominant flexor pollicis brevis (FPB) muscle using silver/silver surface electrodes (surface area $263 \mathrm{~mm}^{2}$; AMBU, Ballerup, Denmark) mounted in bipolar belly-tendon technique. Data was bandpass filtered (20 to 2,000 Hz) and amplified using an Ekida DC universal amplifier (Ekida, Helmstadt, Germany) connected to a Micro $1401 \mathrm{mkII}$ data acquisition unit (Cambridge Electronic Design, Cambridge, UK) with a sampling rate of $5 \mathrm{kHz}$ and stored on a personal computer for online visual display and later offline analysis using Signal software version 5 (Cambridge Electronic Design, Cambridge, UK).

After the subjects were seated comfortably in an armchair, they were asked to rest the stimulating hand on a cushion. Each experimental block started with searching the M1-HAND hotspot (area where the measured MEP is highest at slightly supra-threshold intensities). Thereby, the coil was positioned tangentially to the skull. Being able to recover the hotspot area, it was marked with a small dot on the scalp. After that the resting motor threshold (RMT) as well as active motor threshold (AMT) was determined by using a maximum likelihood threshold hunting procedure [19] with the TMS Motor Threshold Assessment Tool (MTAT 2.0) available from Awiszus et al. [20]. As an cutoff value, a peak-to-peak MEP amplitude of $0.05 \mathrm{mV}$ was chosen.

Later on, for a technical contemplation of the devices, the determined AMTs were taken as a basis to release qTBS protocols without treating subjects, but to check energy losses and coil temperature rise during protocol execution.

\section{Results}

For the biphasic qTBS device we determined the RMT to 46.5 $\pm 12.7 \% \mathrm{MSO}$ and AMT to $34 \pm 6,7 \% \mathrm{MSO}$, whereas the RMT with polyphasic pulses was at $73.5 \pm 9.5 \% \mathrm{MSO}$ and AMT at $51,5 \pm 6,2 \%$ MSO.

\section{Technical considerations}

We aim qTBS protocols to be applied at subthreshold level. As a first guess we took 90 percent of the measured AMT to be the stimulation intensity for the qTBS protocol. This would lead to a level of $30.5 \pm 6.1 \%$ MSO for the biphasic device and at $46.5 \pm 5.4 \%$ MSO for the polyphasic device, respectively.

Based on these mean intensities, the pulse energy of a biphasic and a polyphasic stimulus, as well as the energy loss per pulse was determined. Additionally, we applied a qTBS protocol into air to determine coil warming. Detailed parameters of the applied qTBS protocol are listed in Table 3, row 1 (ISI $1.5 \mathrm{~ms}$, IBI $200 \mathrm{~ms}, 1,440$ pulses, 72 s). Figure 4 displays the coil current as well as the pulse capacitor voltage for one qadripulse of the biphasic and the polyphasic device. Furthermore Table 4 shows all additional device parameters.
Table 4. Device Parameters During the Applied qTBS Protocol

\begin{tabular}{|c|c|c|}
\hline & Biphasic qTBS & Polyphasic \\
\cline { 3 - 3 } & Device & qTBS Device \\
\hline Pulse Capacitor & $66 \mu \mathrm{F}$ & $13.6 \mu \mathrm{F}$ \\
\hline Coil Inductance & $10 \mu \mathrm{H}$ & $10 \mu \mathrm{H}$ \\
\hline Resonant Frequency & $6.2 \mathrm{kHz}$ & $13.7 \mathrm{kHz}$ \\
\hline Mean applied Stimulus Intensity & $30.5 \% \mathrm{MSO}$ & $46.5 \% \mathrm{MSO}$ \\
\hline Initial Pulse Capacitor Voltage & $0.72 \mathrm{kV}$ & $1.31 \mathrm{kV}$ \\
\hline Peak Coil Current & $1.66 \mathrm{kA}$ & $1.30 \mathrm{kA}$ \\
\hline Peak dI/dt & $70.2 \mathrm{~A} / \mu \mathrm{s}$ & $131 \mathrm{~A} / \mu \mathrm{s}$ \\
\hline Pulse Energy per Stimulus & $17.1 \mathrm{~J}$ & $11.7 \mathrm{~J}$ \\
\hline Energy to be recharged per Stimuli & $6.75 \mathrm{~J}$ & $4.49 \mathrm{~J}$ \\
\hline Coil Temperature Rise (set of & $5.5{ }^{\circ} \mathrm{C}$ & $5.2{ }^{\circ} \mathrm{C}$ \\
\hline 1,440 Stimuli) & & \\
\hline
\end{tabular}

The table shows the coil heating, energy losses and essential device parameters that were identified during the qTBS protocol experiment. Data is based on the mean applied stimulus intensity.

\section{Discussion}

Due to the capability of present commercial TMS devices, monophasic pulse shapes are mainly used for single and low frequency rTMS, whereas biphasic pulses are mainly used for rTMS protocols with higher repetition rates like TBS. Other than biphasic stimulators, two or even four monophasic devices can be connected to work with a single application coil. Thus this set-up is able to apply paired pulses as well as QPS with very low ISI. Our biphasic and polyphasic device is able to overcome the limitations of biphasic devices. Thus, it is able to set QPS and qTBS stimuli down to an ISI of $1.0 \mathrm{~ms}$. Due to this fact, known QPS protocols can be retested with biphasic as well as polyphasic stimuli and completely new qTBS protocols with ultra-high repetition rates can be developed.

\section{Experiments}

In a first experiment, we determined MEP with biphasic and polyphasic current waves. Further on we used the measured AMTs to check whether the devices are strong enough to apply QPS and qTBS protocols to subjects in experimental set-ups. Data show that both devices seem strong enough to clearly excite MEPs. Furthermore we observed that coil heating isn't a limiting factor for the introduced ultra-high frequency protocols like qTBS. For the fixed set of protocols listed in Table 3, the applied protocol illustrates worst case as ISI and IBI are shortest, but even there coil temperature only raised less than 6 degree Celsius.

With a previous version of our biphasic qTBS device, which mainly differs in its control unit software from the ones introduced above, but has almost similar hardware structure, we have already applied qTBS protocols to subjects [21]. We observed an increase in cortico-spinal excitability with qTBS, referring to the concept of LTP-like plasticity $[21,22]$. For the polyphasic qTBS this research is ongoing at the moment.

In case qTBS shows that LTP-like and LTD-like plasticity can easily be set and applied in a short period of time, due to shortened treatment time using ultra-high frequency protocols. These protocols may have strong implications for therapeutic purposes such as rehabilitation after stroke and treatment of depression. But, of course our results are only indications and have to be approved by further studies with higher numbers of participants.

Additionally, different QPS and qTBS protocols have to be tested and compared to each other to determine potential effects on plasticity 

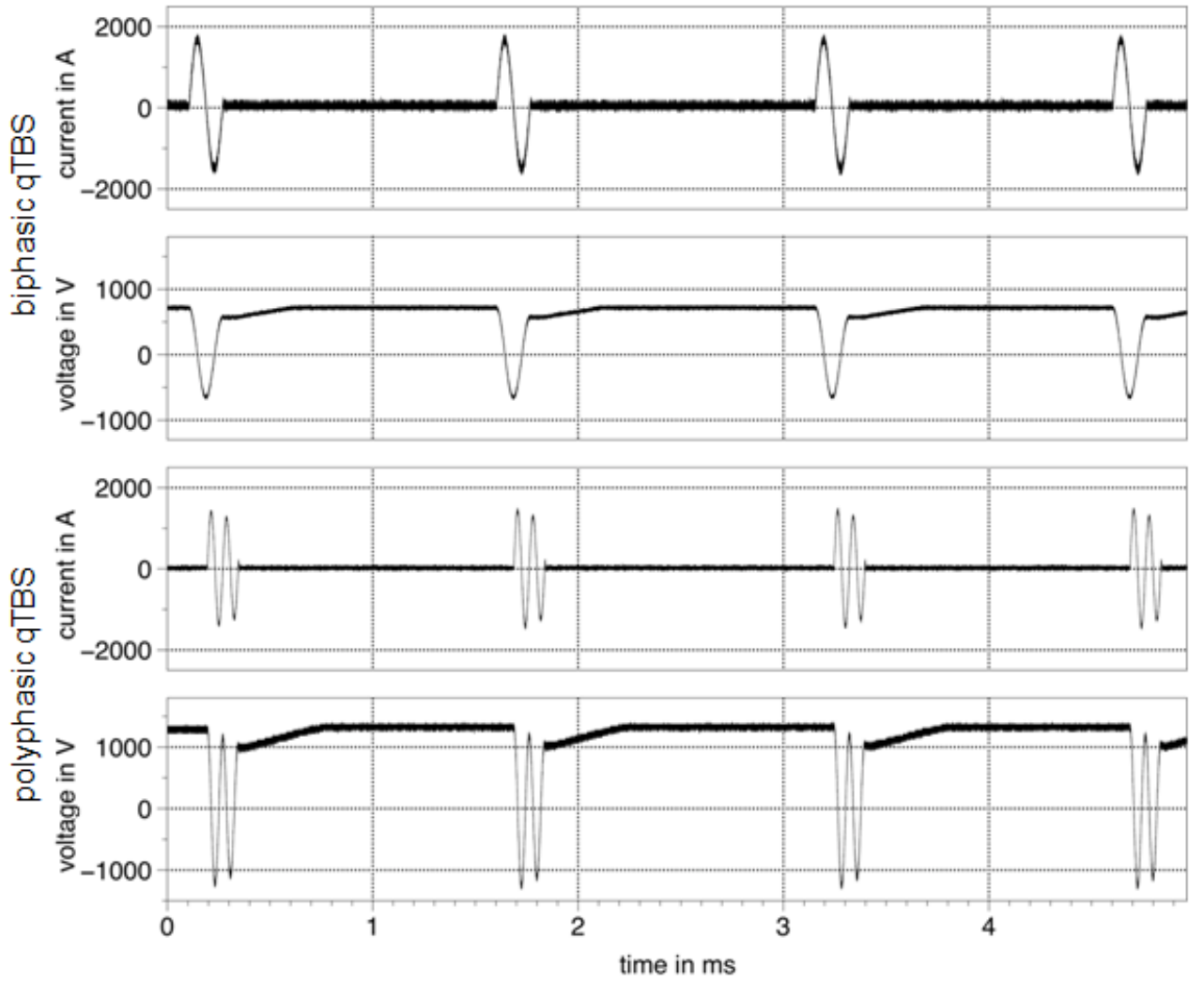

Figure 4. The coil current as well as the pulse capacitor voltage for one qadripulse of the biphasic and the polyphasic device

and to point out the neurophysiological mechanisms underlying the observed effects.

\section{Stimulation device}

To be comparable to at least one commercial TMS device, we included a pulse capacitor in our device that is the same size than used in the PowerMAG device (MAG \& More GmbH,Munich, Germany). Thus the biphasic qTBS device can apply exactly the same biphasic pulses as the PowerMAG (same shape, same duration, same MSO). The presented device can also be used with Magstim stimulation coils (The Magstim Company Ltd, Whitland, UK). However, in this case the fundamental resonant frequency and thus the pulse duration changes.

Davey et al. showed in their computational paper that, relating to membrane voltage, the energy optimal resonant frequency for biphasic stimulation is around $10 \mathrm{kHz}$ [23]. Former research of our working group pointed out that a stimuli consisting of two sine-wave cycles is able to elicit higher MEPs than a biphasic one (single sine-wave) [11]. Combining these two findings led to the construction of the polyphasic QPS device. Due to component availability and mounting space we chose a $13.6 \mu \mathrm{F}$ pulse capacitor. This leads together with the used Figure 8 coil to a fundamental oscillation frequency of $13.7 \mathrm{kHz}$ (resistor losses neglected). In case a Magstim Figure 8 air coil is used (P/N 992500, The Magstim Company Ltd, Whitland, UK) the coil inductance of $17.5 \mu \mathrm{H}$ changes the oscillation frequency to $10.3 \mathrm{kHz}$, which is almost exactly the recommended resonant frequency by Davey et al. [23].

Both devices showed that they clearly can elicit MEPs and that they are able to release quadripulses with high repetition rates. Neither the stimulation coil, nor the power circuitry components inside the device were far from an overheating condition. From an energy consumption point of view, data show that the polyphasic QPS device seems to be more energy efficient than the biphasic one, but to ensure this more subject data are needed. In contrast to this, the biphasic device is stronger when it comes to absolute stimulation strength. This shows the comparison of the percentage levels of MSO at mean qTBS-stimulation level.

\section{Limitations}

We tried to balance easy to use stimulation protocols that led to a fixed set of nine qTBS/QPS-protocols with the possibility to have a maximum amount of flexibility. To get advantage of this flexibility a appendant software has to be used. As a drawback of the possibility to alter almost all protocol parameters, the custom designed protocol has to be chosen very carefully. If not this might lead to stimulation results that might be misinterpreted or even cannot be compared to other studies.

But on the other side, customizing protocols not only by choosing stimulation intensity, but also to individualize protocol parameters might boost stimulation effects or might lead to clearer results between subjects. For instance the ISI might be adjusted to I-wave latencies, which are thought to arise from repeated trans-synaptic firing of pyramidal cells through inter-neuronal networks within the human M1 $[24,25]$. These individual oscillations in turn seem to contribute to and are modulated by plasticity inducing protocols [26]. In addition, I-waves are not only rigid oscillations with timely relation to TMS pulses but the recruitment pattern and latency of these multiple descending volleys underlie inter-individual variability in latencies of around $1.5 \mathrm{~ms}[24,27]$.

Our biphasic qTBS device uses well established sinusoidal full sine coil currents. When it comes to the polyphasic qTBS device, also sinusoidal coil current flow is used, but these double-sine pulses are nowadays not used in commercially available devices. In contrast to e.g. 
cTMS3 that is able to alter pulse shape easily, the pulse shape cannot be altered in our case [12]. But we offer a device type, not taller than today commercially available biphasic stimulation devices, that allow ultra-high frequency (qTBS) protocols with an IBI down to $200 \mathrm{~ms}$. Peterchev et al. describe that quadripulse protocols (QPS) are possible with cTMS3, but unfortunately they do not mention a repetition rate or possible IBI-time in their paper [12].

\section{Safety}

In contrast to monophasic QPS protocols, in which one quadripulse is given every 5 seconds or even less [3], biphasic and polyphasic qTBS can be delivered with the above-presented devices up to a repetiton rate of 5 quadripulses per second. For conducting this ultra-high frequency protocols possible neuro-modulating effects are completely unknown. To reduce the risk of seizure induction, we recommend to apply qTBS very carefully and always on a sub-threshold level. But as there is no knowledge base if stimuli given at this high repetition rates increases seizure risk, shows homoeostatic behavior with minima and maxima, or even affects it at all. Because of that adequate monitoring in our view is compulsory.

\section{Conclusion}

We introduced a circuit topology that is able to apply up to four sinusoidal stimuli with an ISI down to $1.0 \mathrm{~ms}$ and at very high repetition rates up to 5 quadripulses per second (IBI down to 200 $\mathrm{ms})$. These qTBS protocols can be released with a single TMS device not larger than conventional rTMS devices. The presented circuit topology can be used building a biphasic and polyphasic (two sinewaves) stimulation device. Compared to classical monophasic QPS, the presented device enables high repetition rates with which the treatment time might be considerably shortened. For instance, stimulation period of the qTBS protocol used in the experiment set-up was 72 seconds. Furthermore the presented devices allow adaption of the protocols to an individual subject. By setting the ISI time to afore measured I-wave latency might boost the change in neural plasticity. Protocol timings like IBI can be modified during its application by using a trigger-In / trigger-Out functionality. This makes the device interesting for closed loop experiment set-up.

\section{Acknowledgment}

We would like to thank H. R. Siebner for discussions on the device implementation and C. Rümenapp for proofreading of the manuscript.

\section{Funding}

This work was supported in part by Bayerische Forschungsstiftung under Grant AZ 742/02 and in part by the seed funding of Munich School of Bioengineering (MSB), Technical University of Munich. Asterisk indicates corresponding author.

\section{References}

1. Huang YZ, Edwards MJ, Rounis E, Bhatia KP, Rothwell JC (2005) Theta burst stimulation of the human cortex. Neuron 45: 201 - 206

2. Rossini PM, Burke D, Chen R, Cohen LG, Daskalakis Z, et al. (2015) Non-invasive electrical and magnetic stimulation of the brain, spinal cord, roots and peripheral nerves: Basic principles and procedures for routine clinical and research application. An updated report from an I.F.C.N. Committee Clin Neurophysiol 126: 1071-1107 [Crossref]

3. Hamada M, Terao Y, Hanajima R, Shirota Y, Nakatani-Enomoto S, et al. (2008) Bidirectional long-term motor cortical plasticity and meta-plasticity induced by quadropulsetranscranial magnetic stimulation. J Physiol 586: 3927 - 3947.

4. Barker AT, Jalinous R, Freeston IL (1985) Non-invasive magnetic stimulation of human motor cortex. Lancet 1: 1106-1107
5. Cadwell JA, Method and apparatus for magnetically stimulating neurons. U.S. Patent 4940 453, July 10, 1990

6. Nakao K, Matsuyama K, Matsuki N, Ikegaya Y (2004) Amygdala stimulation modulates hippocampal synaptic plasticity. Proc Natl Acad Sci USA 101: 14270 - 14275.

7. Nakamura K, Groiss SJ, Hamada M, Enomoto H, Kadowaki S, et al. (2016) Variability in Response to Quadripulse Stimulation of the Motor Cortex. Brain Stimul 9: 859-866 [Crossref]

8. Di Lazzaro V, Oliviero A, Pilato F, Saturno E, Dileone M, et al (2004) The physiological basis of transcranial motor cortex stimulation in conscious humans. Clin Neurophysiol 115: 255 - 266. [Crossref]

9. Pell GS, Roth Y, Zangen A (2011) Modulation of cortical excitability induced by repetitive transcranial magnetic stimulation: influence of timing and geometrical parameters and underlying mechanisms. Prog Neurobiol 93: 59 - 98. [Crossref]

10. Rothkegel H, Sommer M, Paulus W, Lang N (2010) Impact of pulse duration in single pulse TMS. Clin Neurophysiol 121: 1915-1921. [Crossref]

11. Pechmann A, Delvendah I, Bergmann TO, Ritter C, Hartwigsen G, et al. (2012) The number of full-sine cycles per pulse influences the efficacy of multi-cycle transcranial magnetic stimulation. Brain Stimulation 5: $148-154$

12. Peterchev AV, Jalinous R, Lisanby SH (2008) A transcranial magnetic stimulator inducing near-rectangular pulses with controllable pulse width (cTMS). IEEE Trans Biomed Eng 55: 257 - 266. [Crossref]

13. Peterchev AV, D’Ostilio K, Rothwell JC, Murphy DL. (2014) Controllable pulse parameter transcranial magnetic stimulator with enhanced circuit topology and pulse shaping. J Neural Eng. 11: 056023. [Crossref]

14. Gattinger N, Moessnang G, Gleich B (2012) FlexTMS - A novel repetitive transcranial magnetic stimulation device with freely programmable stimulus currents. IEEE Trans Biomed Eng 5: 1962-1970. [Crossref]

15. Feldman DE (2012) The spike-timing dependence of plasticity. Neuron 75: 556-571

16. Johnson MD, Lim HH, Netoff TI, Connolly AT, Johnson N, et.al. (2013) Neuromodulation for brain disorders: challenges and opportunities. Trans Biomed Eng 6: 610-624. [Crossref]

17. Lewis PM, Thomson RH, Thomson JV, Rosenfeld JV, Fitzgerald PB. (2016) Brain Neuromodulation Techniques: A Review. Neuroscientist 22: 406-421.

18. Rossi S, Hallett M, Rossini PM, Pascual-Leone A, et al. (2009) Safety, ethical considerations, and application guidelines for the use of transcranial magnetic stimulation in clinical practice and research. Clin.Neurophysiol 120: 2008 - 2039 [Crossref]

19. Awiszus F, Feistner H (1995) Rapid on-line estimation of response to transcranial magnetic and peripheral nerve electrical stimulation in single human motoneurons. $J$ Neurosci Methods 61: 91 - 97. [Crossref]

20. Awiszus F, Borckardt JJ (2016) TMS Motor Threshold Assessment Tool

21. Jung NH, Gleich B, Gattinger N, Hoess C, Haug C, et al. (2016) Quadri-Pulse Theta Burst Stimulation using Ultra-High Frequency Bursts - A New Protocol to Induce Changes in Cortico-Spinal Excitability in Human Motor Cortex. PLOS ONE 11 e0168410. [Crossref]

22. Bliss TV, Lomo T (1973) Long-lasting potentiation of synaptic transmission in the dentate area of the anaesthetized rabbit following stimulation of the perforant path. $J$ Physiol 232: 331 - 356. [Crossref]

23. [crossref] Davey K1, Epstein CM (2000) Magnetic stimulation coil and circuit design. IEEE Trans Biomed Eng 47: 1493-1499.

24. Di Lazzaro V, Profice P, Ranieri F, Capone F, Dileone M, et al. (2012) I-wave origin and modulation. Brain Stimulation 5: 512 - 525. [Crossref]

25. Rusu CV, Murakami M, Ziemann U, Triesch J (2014) A model of TMS-induced I-waves in motor cortex. Brain Stimulation 7: 401 - 414. [Crossref]

26. Hamada M, Murase N, Hasan A, Balaratnam M, Rothwell JC (2013). The role of interneuron networks in driving human motor cortical plasticity. Cereb Cortex 23: 1593-1605. [Crossref]

27. Delvendahl I, Gattinger N, Berger T, Gleich B, Siebner HR (2014) The role of pulse shape in motor cortex transcranial magnetic stimulation using full-sine stimuli. PLOS ONE 9: e115247 [Crossref]

Copyright: (C2018 Gattinger N. This is an open-access article distributed under the terms of the Creative Commons Attribution License, which permits unrestricted use, distribution, and reproduction in any medium, provided the original author and source are credited. 